\title{
Current management of acute scaphoid fractures: a review
}

\author{
Jason PY Cheung *, Chris YK Tang, Boris KK Fung
}

\section{A B S T R A C T}

The aim of this review was to present currently available evidence on the management of acute scaphoid fractures. Acute scaphoid fractures are usually diagnosed by a combination of history, physical examination, and radiography. However, in many patients scaphoid fractures are still missed. Thus, the general trend is to over-treat patients with

This article was published on 9 December 2013 at www.hkmj.org. a suspicion of scaphoid fracture. Many aspects of scaphoid fracture management are still controversial and different institutions vary in their approach.
Hong Kong Med J 2014;20:52-8

DOI: $10.12809 / \mathrm{hkmj} 134146$

JPY Cheung *, MB, BS, MMedSC

CYK Tang, $M B, B S$

BKK Fung, FRCS, FCRCE (Ortho)

Department of Orthopaedics and Traumatology, University of Hong Kong Medical Centre, Queen Mary Hospital, 102 Pokfulam Road, Hong Kong

* Corresponding author: jcheung98@hotmail.com

\section{Introduction}

Scaphoid fractures have been extensively investigated in the past. They are the most common type of carpal fractures and are usually found in young men, ${ }^{1}$ accounting for 2 to $7 \%$ of all fractures, and 70 to $80 \%$ of carpal fractures. ${ }^{2}$ Scaphoid fractures affecting the waist $(70 \%)$ are the commonest type in adults, followed by distal pole fractures (10-20\%), proximal pole fractures (5-10\%), and tubercle fractures (5\%). ${ }^{2}$ Conversely, $52 \%$ of all scaphoid fractures in children involve the tubercle, 33\% affect the distal third, and $15 \%$ affect the waist, ${ }^{2}$ though this discrepancy may be partially accounted by the fact that children's scaphoids are not fully ossified in their proximal pole, making a waist fracture look like a proximal pole fracture. Scaphoid fractures are commonly prone to complications due to delayed treatment or misdiagnosis. Avascular necrosis is particularly common, with estimated rates of 13 to $50 \%{ }^{3}$ Other complications such as nonunion, malunion, carpal instability, and radiocarpal arthrosis are also frequently seen. Thus, early diagnosis and treatment are critical for a better prognosis.

Acute scaphoid fractures can be difficult to diagnose. According to some studies, the prevalence of true fractures among patients with suspected scaphoid fractures may only be 5 to $10 \% .{ }^{1}$ Multiple radiographs may not be able to pick up all scaphoid fractures and consequently, clinicians attempt to avoid undertreatment by liberal use of cast immobilisation. However, overtreating patients results in a loss of work days and productivity and increased health care costs, ${ }^{1}$ and it is conjectured that 76 to $100 \%$ of such cases undergo inappropriate initial immobilisation. ${ }^{4}$

Undisplaced scaphoid fractures are not benign injuries and warrant prolonged plaster cast immobilisation or early osteosynthesis. Clinicians should have a high index of suspicion and must be meticulous in studying the clinical examination findings and radiographs.

\section{Anatomy}

The scaphoid forms the bridge to the distal carpal row (trapezium, trapezoid, and capitates) and the proximal row (proximal pole to the lunate) by a ligamentous network including the scapholunate interosseous ligament and extrinsic palmar ligaments. The scaphoid can be simply divided anatomically into the proximal pole, the waist, and the distal pole. Its surface is mostly ( $80 \%)$ covered by articular cartilage. ${ }^{5}$ The articular surfaces include the proximal pole articulation with the radius and lunate and the distal pole articulation with the capitate, trapezium, and trapezoid.

Being predominantly articular, the blood supply to the scaphoid has limited access. Branches from the radial artery (dorsoradial arteries) form arches at the dorsal wrist capsule and enter the scaphoid at its dorsal ridge. This provides 70 to $80 \%$ of the blood supply to the scaphoid. ${ }^{3,5}$ The other 20 to $30 \%$ are supplied by the superficial palmar arch or branches of the radial artery that reach the distal palmar area of the scaphoid. ${ }^{3,5}$ Thus, the proximal pole depends solely on intraosseous blood flow and fractures here have a high risk of osteonecrosis associated with a prolonged period of healing. An average of 3 to 6 months are required for healing in these fracture types and nonunion is quoted to ensue in 5 to $10 \% .^{3}$ Additional nutrient arteries supply the distal pole via the area of the scaphotrapezium ligamentous attachment.

\section{Biomechanics}

The usual mechanism of injury is a forced dorsiflexion 
wrist injury such as a fall on outstretched hand. Cadaveric studies have shown that fractures occur when the wrist is kept in 95 to 100 degrees of extension and a dorsiflexion load is applied to the radial half of the wrist with the radioscaphocapitate ligament kept as the fulcrum. ${ }^{5}$ Failure in compression occurs on the dorsal side of the bone and failure in tension on the palmar side. Dorsal angulation of the fracture is caused by opposing rotational moments on the proximal and distal poles of the scaphoid. Furthermore, dorsal intercalated segmental instability (DISI) ensues if the proximal carpal row is in extension. Bending forces to wrist fractures are resisted by intact scaphoid-carpal ligaments. ${ }^{6}$ Distal pole and tubercle fractures are due to direct impact and forced ulnar deviation causes avulsion fractures at radial collateral ligament attachments.

\section{Assessment}

Classical examination findings of tenderness at the anatomic snuffbox and the volar aspect of the distal tuberosity and positive scaphoid compression test (pain on axial compression of the thumb metacarpal) raise suspicions that warrant further investigation. A study by Unay et $\mathrm{al}^{7}$ suggested that pain during thumb-index pinching (sensitivity $73 \%$, specificity $75 \%$, positive predictive value $96 \%$, and negative predictive value 23\%) and pain during forearm pronation (sensitivity $79 \%$, specificity $58 \%$, positive predictive value $82 \%$, and negative predictive value $54 \%$ ) aid the diagnosis of scaphoid fractures, but are absent in $27 \%$ of cases. Overall, the specificity for clinical examination shown in the literature was only 74 to $80 \%^{3}$ and the mean positive predictive value was quoted to be only $21 \% .^{8}$ Other physical findings that may help to diagnose scaphoid fractures include limitation in end arc of motion with flexion and radial deviation, and reduced grip strength.

Nonunion can be found in up to $12 \%$ in cases of missed scaphoid fractures. ${ }^{3}$ Therefore, scaphoid fractures must be identified early and immobilised appropriately. Imaging techniques can aid in the diagnosis of occult fractures. Plain film radiography can detect a fracture in 70 to $90 \%$ of cases. ${ }^{8}$ Four views are necessary: posterior-anterior (PA) wrist, lateral wrist with extended fingers, anterior-posterior (AP) wrist with flexed fingers (scaphoid lies parallel to the film with flexed fingers), and the wrist in 25 to 45 degrees supination with flexed fingers. ${ }^{9}$ Neutral or ulnar-deviated PA films do not show waist fractures well, because the axis of the scaphoid is flexed towards the beam and the tubercle overhangs the body. Fractures of the dorsal sulcus are best demonstrated on a 45-degree PA oblique view, and proximal pole fractures on a 45 -degree AP oblique view. ${ }^{2}$ Scaphoid waist fractures are best seen on an ulnar-deviated PA view with 20 degrees of elbow flexion. ${ }^{2}$ The semipronated oblique view visualises the waist of the

\section{舟狀骨骨折治療的回顧研究 鍾培言、鄧育昀、馮國強}

本研究綜合現有文獻對於舟狀骨骨折治療的方向。診斷舟狀骨骨折的 方法包括病史、臨床檢查及X光檢查。可惜仍然有很多不能被正確診 治的舟狀骨骨折病例。因此, 現代舟狀骨骨折治療方法傾向對懷疑個 案也作出治療。舟狀骨骨折治療的許多方面仍具爭議性, 而每所醫院 對舟狀骨骨折治療都有不同的看法

scaphoid best, but multiple views such as PA, lateral and ulnar-deviated and clenched-fist views may be required to make a correct diagnosis. ${ }^{9}$ Lateral X-rays may only detect tuberosity and distal third fractures, ${ }^{10}$ but are also essential to show the carpal alignment and distal radioulnar joint alignment. A proper view should show a co-linear capitate and radius, with the pisiform located between the distal pole of the scaphoid and the body of the capitate. ${ }^{3}$

Other adjuvant imaging techniques may be required to diagnose scaphoid fractures. Computed tomography (CT) is usually used to identify fractures and nonunions and for preoperative planning, and it is better for detecting occult fractures of the cortex with a mean sensitivity of $94 \%$ and specificity of $96 \%{ }^{1}$ It was found to have a mean negative predictive value of $99 \%$ in a study by Ty et $\mathrm{al}^{11}{ }^{11}$ which means it is very unlikely to miss a scaphoid fracture. Furthermore, CT is readily available in urgent care settings and is more cost-effective than magnetic resonance imaging (MRI).

Magnetic resonance imaging has a mean sensitivity of $98 \%$ and specificity of $99 \% .{ }^{1}$ It can locate trabecular fractures and help identify other causes of wrist pain if a fracture is not found, besides helping to determine the vascularity of the proximal pole preoperatively. It is especially useful in diagnosing proximal pole fractures, which may develop avascular necrosis. Acute fractures show normal or decreased $\mathrm{T} 1$ and increased T2 intensity. ${ }^{3}$ Nonunion and impaired vascularity are often seen with low T1 and T2 marrow signal intensity which correlates with poor healing. ${ }^{3}$ Notably, MRI is more sensitive in detecting occult scaphoid fractures, with fewer falsepositives than bone scans. ${ }^{3}$ Thus, it can accurately exclude patients without scaphoid fractures and facilitate discontinuing immobilisation. For planning the management of cases of scaphoid nonunion, MRI can be used following internal fixation, as the bone marrow signal can be assessed even in the presence of a titanium alloy screw. ${ }^{12}$

Scaphoid fractures are commonly associated with injuries to the carpal ligament or triangular fibrocartilage complex, and reported in 35\% of affected patients and intercarpal soft tissue injury may ensue 
in $86 \%$ of instances. ${ }^{13}$ Usually these conditions can be treated conservatively as mild ligament tears heal without long-term complications. However, carpal ligament injuries may lead to symptomatic chronic carpal instability. Thus, surgical fixation and early mobilisation may be indicated in more severe cases. In the most serious cases, scaphoid fractures can constitute part of the abnormality in perilunate or lunate dislocations of the wrist. Identifying and assessment of these injuries can be performed with a wrist arthrogram or arthroscopy. Besides soft tissue injuries, distal radial fractures are also found quite commonly due to shared mechanisms of injury.

\section{Classification}

Herbert's classification system is the most wellknown and commonly used, as it defines stable and unstable fractures. Type A fractures are stable acute fractures; type B are unstable acute fractures; type $\mathrm{C}$ are delayed unions ( $>6$ weeks of plaster immobilisation), and type $D$ are established nonunions (fibrous or sclerotic). Stable fractures include fractures of the tubercle (A1) and incomplete fracture of the waist (A2). Type B fractures are acute unstable fractures. These include subtypes B1 (oblique fractures of the distal third of the scaphoid), B2 (displaced or mobile complete fractures of the waist), B3 (proximal pole fractures), B4 (fracture dislocations), and B5 (comminuted fractures). ${ }^{14}$

\section{Treatment}

Treatment of acute scaphoid fractures is controversial and each centre has different criteria for conservative versus operative treatment. In patients with a suspected fracture but no obvious findings on X-rays, most centres advocate joint immobilisation before repeat imaging for reassessment at a later time. However, casting makes exclusion of fracture and determination of fracture union by follow-up X-rays more difficult, for which reason follow-up CT may be warranted. Fixation is suggested if the MRI shows a proximal pole fracture. ${ }^{3}$ Since cartilage covers $80 \%$ of the scaphoid, no fracture callus can be made to stabilise the fracture site during healing ${ }^{6}$ and thus rigid fixation is mandatory. Healing on the X-ray is inferred from disappearance of the fracture line, spot welding between fracture fragments, or callus formation. With these findings, immobilisation can be discontinued and patients can be allowed a gradual return to activities.

Treatment depends primarily on the location and degree of displacement. Distal pole fractures are usually due to avulsion of the scaphoid tuberosity or impaction of the distal articular surface. These fractures have a good vascular supply that enables rapid healing in 4 to 6 weeks with a short arm thumb spica cast. ${ }^{5}$ Traditionally, undisplaced, stable waist fractures are treated in short- or long-arm casts.
These often involve prolonged immobilisation of up to 12 weeks. $^{3}$ Union can be achieved in greater than $90 \%$ of affected individuals. ${ }^{5}$ However, recent evidence suggests improved results with operative fixation. Prolonged immobilisation disrupts collagen homeostasis resulting in loss of normal connective tissue characteristics, allowing tendons to glide and the joint capsule to stretch.

\section{Non-operative}

Some studies suggest a further 6 weeks of immobilisation before offering operative fixation if at 6 weeks the CT shows an unhealed fracture. ${ }^{3}$ Others suggest that most fractures of the scaphoid waist unite after 8 weeks of immobilisation but may require as long as 12 weeks. ${ }^{15}$ Overall scaphoid waist fractures can unite satisfactorily in 85 to $95 \%$ of patients. ${ }^{16}$ Scaphoid fractures are generally immobilised in a scaphoid cast (proximal phalanx of thumb in palmar abduction leaving the interphalangeal joint free) or a Colles' cast (exposing the thenar eminence and leaving the metacarpophalangeal joint free). Pinch grip function is impaired in scaphoid casts; the Colles' cast allows for greater overall range of thumb movement and improved function. ${ }^{17}$ The fine pinch grip of the thumb should be preserved as much as possible, for which Colles' casting rather than scaphoid casting offers better preservation of function. ${ }^{17}$ However, there are no data on the longterm results of fracture healing following these two types of casting.

The wrist position during immobilisation has also been investigated. Hambidge et $\mathrm{a}^{18}$ showed that the frequency of nonunion was not influenced by the position of immobilisation $(\mathrm{P}=0.46)$ and 108 of 121 fractures united after 12 weeks of immobilisation. However, wrists immobilised in 20-degree flexion results in less extension at the 6-month follow-up. ${ }^{18}$ A possible explanation was that immobilisation in flexion may have produced increased flexion stress on the fracture, causing a humpback deformity, which restricts wrist extension and causes persistent pain..$^{18}$ An alternative explanation for restricted extension could be related to soft-tissue injury, joint adhesions, or contracture of the palmar capsule at the wrist. ${ }^{18}$ Immobilisation in a Colles' cast with the wrist in 20degree extension is therefore recommended. ${ }^{18}$

Long-arm versus short-arm casting is controversial. Biomechanical studies in cadavers show fracture site motion during forearm rotation. ${ }^{5}$ Forearm rotation leads to excessive scaphoid fracture motion, which may impair bone healing. Excessive motion is an indication for long-arm casting to restrict forearm rotation and to reduce the associated displacement of bone fragments. Displacement of more than $1 \mathrm{~mm}$ is associated with instability and is an indication for open reduction and internal fixation. ${ }^{19}$ A cadaveric study 
on scaphoid waist fractures by Kaneshiro et $\mathrm{al}^{20}$ showed that significant fracture site motion could occur with forearm rotation in a short-arm thumb spica cast. Some forearm rotation may even occur when long-arm casting is used, but the displacement should be less than $0.5 \mathrm{~mm} .{ }^{20}$ Thus, long-arm casting is recommended.

As mentioned above, most studies suggest immobilisation for 8 to 12 weeks for scaphoid waist fractures. ${ }^{15-18,21}$ Geoghegan et $\mathrm{al}^{15}$ showed that $89 \%$ of undisplaced scaphoid fractures achieved union by 4 weeks and mobilisation could begin at that time. Allowing mobilisation with a wrist splint at week 4 can reduce the period of disability associated with nonoperative treatment. ${ }^{15}$ Böhler et $\mathrm{al}^{9}$ showed a $96 \%$ healing rate in 580 undisplaced scaphoid waist fractures with 6 weeks of immobilisation with a simple unpadded dorsal fist plaster splint that includes the thumb. The hand needs to be kept in neutral position when using the dorsal plaster splint. ${ }^{9}$

Nonoperative treatment is successful in achieving union but there are disadvantages of immobilisation, namely stiffness, diminished grip strength, and delayed return to work. Pseudoarthrosis ensues in approximately $4 \%$ of patients who only have casting, and is usually associated with vertical oblique fracture patterns (due to tilting and shearing forces) and diastasis between bone fragments. ${ }^{9}$ Young and active patients are unlikely to tolerate several months of immobilisation due to the pressures of work or athletics. Currently, therefore, there is a trend towards operative management to reduce the number of days of inactivity.

\section{Operative}

In theory, early internal fixation has the benefits of early return of wrist movement, a higher rate of union, an early return to work and sport, and avoiding the need for a plaster cast. Reduction of the fracture in anatomical alignment is vital for good results. If reduction cannot be achieved by closed means, open reduction is necessary. Usually simple hyperextension of the wrist can achieve good reduction. In addition, Moser et $\mathrm{al}^{22}$ suggested having the arm in extension during surgery to maintain the reduction. Percutaneous fixation of the fracture limits the risk of devascularising fracture fragments and protects the ligaments and volar capsule. However, for percutaneous fixation to be feasible, the fracture must not be displaced or reducible by closed means. On the contrary, there is no controversy about treating displaced scaphoid fractures by open reduction and internal fixation. ${ }^{5}$ Surgical stabilisation allows the patient to perform early range of motion exercises and avoids prolonged immobilisation.

In the literature, evidence in favour of surgery is not overwhelming. A meta-analysis by Bhandari and Hanson ${ }^{23}$ showed that internal fixation resulted in a significantly earlier return to work (by 8 weeks) as compared with casting. However, both methods did not differ in terms of outcomes such as grip strength $(\mathrm{P}=0.24)$ and range of motion $(\mathrm{P}=0.67) .{ }^{23}$ Furthermore, the risk of nonunion was also found to be similar $(\mathrm{P}=0.28) .{ }^{23}$ Similarly, Saedén et $\mathrm{al}^{21}$ showed that a follow-up period of up to 12 years after the fracture revealed no difference in pain or discomfort between the operative and conservative treatments. Dias et $\mathrm{al}^{24}$ had similar results in terms of grip strength and range of movement after follow-up for 93 months. However, McQueen et $\mathrm{al}^{25}$ showed that percutaneous screw fixation attained quicker union (9 vs 14 weeks, $\mathrm{P}<0.001$ ) for treatment of Herbert types B1 and B2 fractures of the scaphoid waist.

The two usual approaches for percutaneous fixation of scaphoid fractures involve volar traction assistance and the dorsal minimal incision (manual reduction with a guidewire as a joystick technique or arthroscopy-assisted reduction). In the volar technique, the wrist is extended over a towel roll to allow proper insertion of the guidewire. Yip et $\mathrm{al}^{26}$ suggested the 45-degree supination oblique view when determining the length of the screw and avoiding over-penetration into the radioscaphoid joint space. The headless screw must be fully buried beneath the articular cartilage of the proximal scaphoid, so as to avoid radioscaphoid impingement. Scaphoid fixation is best accomplished with the longest screw placed in the distal scaphoid poles. ${ }^{6}$ Bone density is greatest in the scaphoid poles, where it provides the best fixation. ${ }^{6}$ Fractures of the distal two thirds can also be approached volarly, as this approach avoids injury to the dorsal blood supply. The volar technique is contra-indicated in proximal pole and oblique fractures, as the screw cannot cross the fracture line perpendicularly to obtain adequate compression and purchase. ${ }^{27}$ This leads to displacement of the fracture. During surgery, the scaphoid is in a flexed posture relative to the longitudinal alignment of the distal radius. From the volar approach, the proximal point to aim at is the proximal ulnar corner of the scaphoid at the insertion of the scapholunate ligament. ${ }^{28}$ The starting point of the surgery is at the scaphotrapezial joint through the proximal thenar muscles. Besides a small terminal branch of the radial nerve, the operation is at a safe distance from the median nerve motor branch and from the radial artery. The drawback of volar surgical approaches is the difficulty in obtaining fracture reduction, which may therefore result in nonunion of proximal scaphoid pole fractures. ${ }^{6}$ The trapezium is in a position that blocks wire placement volarly, and therefore placing a guidewire along the central scaphoid axis is difficult such that the screw can also penetrate the joint. ${ }^{6}$

For the dorsal approach, the distal point aimed 
at is the centre of the scaphotrapezial joint or the base of the thumb. Thus, this allows for a more central placement in the distal pole. ${ }^{28}$ The dorsal approach provides direct unobstructed access to the proximal pole permitting the placement of a central axis guidewire for screw implantation. There is better fracture fixation as the purchase of the screw threads in the proximal fragment tends to be greater. ${ }^{29}$ However, the disadvantages of this technique include poor exposure to the distal third of the scaphoid, damage to the articular cartilage of the proximal pole of scaphoid, potential entrapment of the extensor tendons, damage to the dorsal ligaments, and risk of vascular injury. ${ }^{29,30}$ Moreover, to insert the screw through the most proximal part, the wrist has to be fully flexed during the procedure. ${ }^{29}$ Flexing the wrist may cause the distal fragment to adopt a flexed posture and cause the proximal fragment to follow the lunate into an extended posture, producing the hump-back deformity. ${ }^{29}$ Distal pole fractures can present technical difficulties for insertion of a volar screw perpendicular to the fracture line and are therefore best suited for fixation using the dorsal fixation technique. ${ }^{27}$

Arthroscopy can assist fracture reduction, and real-time mini-fluoroscopy can guide the dorsal percutaneous insertion of a headless compression screw. ${ }^{6}$ Arthroscopic examination can also permit assessment of concurrent ligamentous injuries and demonstrate osteochondral fragments at the midcarpal row. ${ }^{6}$ Thus, arthroscopic-assisted surgery can treat both scaphoid fractures and carpal ligament injuries. $^{31}$ Arthroscopy also preserves the key ligaments and blood supply, allowing for immediate hand rehabilitation. ${ }^{32}$

\section{Complications}

Up to $20 \%$ of patients may endure residual pain despite a normal grip strength and wrist movement after surgery. ${ }^{33}$ These persistent symptoms can be due to intra-operative articular cartilage damage. Damage affects the distal scaphoid-radial styloid joint first, and later progresses to the scaphocapitate and capitolunate joints. Osteoarthritis occurred in $5 \%$ of patients with a history of a scaphoid fracture despite normal healing, ${ }^{34}$ but usually did not present until several decades after injury. The opening of the scaphotrapezial joint for screw insertion during surgery may result in the development of osteoarthritis. ${ }^{21}$

Malunion of the scaphoid may produce a flexion (humpback) deformity with ulnar deviation and pronation of the distal fragment. Flexion deformity within the scaphoid causes loss of extension at the radiocarpal and midcarpal joints. Amadio et $\mathrm{al}^{35}$ showed that only $27 \%$ of patients with interscaphoid angles of $>35$ degrees had satisfactory results in terms of pain, function, movement, and strength. This contrasts with $83 \%$ of the patients having satisfactory results with interscaphoid angles of $<35$ degrees. ${ }^{35}$

It is accepted that for a scaphoid fracture, 6 months must elapse before a diagnosis of nonunion can be made. Such patients endure pain and poor function; in 35\% lateral wrist X-rays yielded a humpback deformity (due to flexion angulation between the proximal and distal scaphoid poles) of patients, ${ }^{4}$ and $42 \%$ showed DISI. ${ }^{4}$ In a review of 104 patients with symptomatic nonunion, all of them developed osteoarthritis. ${ }^{36}$ Fibrous union is visualised as irregularity at the fracture line, while in pseudoarthrosis the two bone halves move independently causing articular damage to the radial facet. ${ }^{33}$ Displacement of the nonunion with the incongruent cartilaginous surfaces and carpal instability also contributes to the development of osteoarthritis. ${ }^{4}$ Many now advocate internal fixation and the use of bone grafting for the treatment of established nonunion. Bone healing usually occurs (in about $75 \%$ of cases) but there may be persistent humpback deformity (16\%), associated DISI deformity (12\%), and osteonecrosis (4\%). ${ }^{4}$ Nevertheless, late osteoarthritis is inevitable and cannot be avoided, even by this operation. ${ }^{33}$ This is an expected consequence of the disease process due to cartilage destruction. The main predictor of healing or failure was the time elapsed between the initial fracture and the treatment of the established nonunion. A delay of 5 years or more would decrease the success rate to only $62 \%{ }^{4}$

Complications of open repair include hypertrophic scarring, avascular necrosis, carpal instability, donor site pain, bone graft infection, screw protrusion, and reflex sympathetic dystrophy. ${ }^{6,28}$ Due to the cartilaginous surface and fracture healing being an intraosseous process, open fixation of a fractured scaphoid may further jeopardise the blood supply of the scaphoid and drain away the fracture haematoma. ${ }^{26}$ The radiocarpal ligaments may also be damaged during open surgery. ${ }^{26}$ These complications may be avoided using a minimally invasive arthroscopic approach. ${ }^{6}$

\section{Our centre's practice}

When a patient is admitted with the clinical suspicion of a scaphoid fracture (mechanism of injury, tenderness at the anatomical snuffbox) but no obvious fracture line seen on X-rays, we provide the patient with a short-arm scaphoid cast and then order a CT. The CT includes a coronal view to better screen for any fracture. If no fracture is evident, a soft splint is given for pain relief.

For an undisplaced non-comminuted waist fracture, we offer conservative treatment with a short-arm scaphoid cast for 6 weeks with interval $\mathrm{X}$-ray monitoring. Casting is kept for up to 4 to 8 
weeks if the fracture line is still seen before surgery is considered. We rarely perform surgery in this patient group because we attained a $95 \%$ union rate in compliant patients. In distal pole fractures, the healing is generally quite good and we rarely resort to excision.

We recommend surgery for all other types of fracture alignment to prevent nonunion. We generally use Herbert screws or cannulated screws, subject to the surgeon's preference. We almost always use the percutaneous approach unless the reduction cannot be obtained well. We use a volar approach antegrade from the scaphoid tubercle as we try to avoid the dorsal approach to prevent cartilage injury. We, however, do use the dorsal approach for proximal pole fractures. The success rate is about $95 \%$ for percutaneous fixations and 85 to $90 \%$ for open reductions.

For rehabilitation, we allow free mobilisation on postoperative day 1 in waist fractures if the fixation is rigid; we keep a slab for 2 weeks if the fracture fixation is doubtful. We nevertheless allow supervised gentle mobilisation for these fractures. In proximal pole fractures, the purchase is usually doubtful and we immobilise the wrist for 4 weeks.

In cases of nonunion, a plain MRI is helpful for the assessment of avascular necrosis as these conditions may require additional procedures such as grafting. This condition is found in our hospital 12 to 18 times a year, and is always due to late referrals or improper primary management including casting for displaced waist fractures.

\section{Conclusions}

The current literature indicates no standard treatment for scaphoid fractures. Different centres have different approaches to the treatment of scaphoid fractures and the evidence on which they rely is controversial. However, the current trend is to treat scaphoid fractures operatively, so as to limit the number of days away from work and to allow patients to regain function sooner.

\section{References}

1. Yin ZG, Zhang JB, Kan SL, Wang XG. Diagnosing suspected scaphoid fractures: a systematic review and meta-analysis. Clin Orthop Relat Res 2009;468:723-34.

2. Shenoy R, Pillai A, Hadidi M. Scaphoid fractures: variation in radiographic views-a survey of current practice in the West of Scotland region. Eur J Emerg Med 2007;14:2-5.

3. Adams JE, Steinmann SP. Acute scaphoid fractures. Orthop Clin North Am 2007;38:229-35, vi.

4. Schuind F, Haentjens P, Van Innis F, Vander Maren C, Garcia-Elias M, Sennwald G. Prognostic factors in the treatment of carpal scaphoid nonunions. J Hand Surg Am 1999;24:761-76.

5. Puopolo SM, Rettig ME. Management of acute scaphoid fractures. Bull Hosp Jt Dis 2003;61:160-3.

6. Slade JF 3rd, Gillon T. Retrospective review of 234 scaphoid fractures and nonunions treated with arthroscopy for union and complications. Scand J Surg 2008;97:280-9.

7. Unay K, Gokcen B, Ozkan K, et al. Examination tests predictive of bone injury in patients with clinically suspected occult scaphoid fracture. Injury 2009;40:12658.

8. Brookes-Fazakerley SD, Kumar AJ, Oakley J. Survey of the initial management and imaging protocols for occult scaphoid fractures in UK hospitals. Skeletal Radiol 2009;38:1045-8.

9. Böhler L, Trojan E, Jahna $H$. The results of treatment of 734 fresh, simple fractures of the scaphoid. J Hand Surg Br 2003;28:319-31.

10. Cheung GC, Lever CJ, Morris AD. X-ray diagnosis of acute scaphoid fractures. J Hand Surg Br 2006;31:104-9.

11. Ty JM, Lozano-Calderon S, Ring D. Computed tomography for triage of suspected scaphoid fractures. Hand (N Y) 2008;3:155-8.

12. Ganapathi M, Savage R, Jones AR. MRI assessment of the proximal pole of the scaphoid after internal fixation with a titanium alloy Herbert screw. J Hand Surg Br 2001;26:3269.

13. Wong TC, Yip TH, Wu WC. Carpal ligament injuries with acute scaphoid fractures-a combined wrist injury. J Hand Surg Br 2005;30:415-8.

14. Herbert TJ, Fisher WE. Management of the fractured scaphoid using a new bone screw. J Bone Joint Surg Br 1984;66:114-23.

15. Geoghegan JM, Woodruff MJ, Bhatia R, et al. Undisplaced scaphoid waist fractures: is 4 weeks' immobilisation in a below-elbow cast sufficient if a week $4 \mathrm{CT}$ scan suggests fracture union? J Hand Surg Eur Vol 2009;34:631-7.

16. Dias JJ, Wildin CJ, Bhowal B, Thompson JR. Should acute scaphoid fractures be fixed? A randomized controlled trial. J Bone Joint Surg Am 2005;87:2160-8.

17. Karantana A, Downs-Wheeler MJ, Webb K, Pearce CA, Johnson A, Bannister GC. The effects of Scaphoid and Colles casts on hand function. J Hand Surg Br 2006;31:4368.

18. Hambidge JE, Desai VV, Schranz PJ, Compson JP, Davis TR, Barton NJ. Acute fractures of the scaphoid. Treatment by cast immobilisation with the wrist in flexion or extension? J Bone Joint Surg Br 1999;81:91-2.

19. Gelberman RH, Wolock BS, Siegel DB. Fractures and non-unions of the carpal scaphoid. J Bone Joint Surg Am 1989;71:1560-5.

20. Kaneshiro SA, Failla JM, Tashman S. Scaphoid fracture displacement with forearm rotation in a short-arm thumb spica cast. J Hand Surg Am 1999;24:984-91.

21. Saedén B, Törnkvist H, Ponzer S, Höglund M. Fracture of the carpal scaphoid. A prospective, randomised 12-year follow-up comparing operative and conservative treatment. J Bone Joint Surg Br 2001;83:230-4.

22. Moser VL, Krimmer H, Herbert TJ. Minimal invasive treatment for scaphoid fractures using the cannulated herbert screw system. Tech Hand Up Extrem Surg 2003;7:141-6.

23. Bhandari M, Hanson BP. Acute nondisplaced fractures of the scaphoid. J Orthop Trauma 2004;18:253-5.

24. Dias JJ, Dhukaram V, Abhinav A, et al. Clinical and radiological outcome of cast immobilisation versus surgical treatment of acute scaphoid fractures at a mean follow-up of 93 months. J Bone Joint Surg Br 2008;90:899-905.

25. McQueen MM, Gelbke MK, Wakefield A, Will EM, 
Gaebler C. Percutaneous screw fixation versus conservative treatment for fractures of the waist of the scaphoid: a prospective randomised study. J Bone Joint Surg Br 2008;90:66-71.

26. Yip HS, Wu WC, Chang RY, So TY. Percutaneous cannulated screw fixation of acute scaphoid waist fracture. J Hand Surg Br 2002;27:42-6.

27. Shin AY, Hofmeister EP. Percutaneous fixation of stable scaphoid fractures. Tech Hand Up Extrem Surg 2004;8:8794.

28. Naranje S, Kotwal PP, Shamshery P, Gupta V, Nag HL. Percutaneous fixation of selected scaphoid fractures by dorsal approach. Int Orthop 2010;34:997-1003.

29. Wu WC. Percutaneous cannulated screw fixation of acute scaphoid fractures. Hand Surg 2002;7:271-8.

30. Polsky MB, Kozin SH, Porter ST, Thoder JJ. Scaphoid fractures: dorsal versus volar approach. Orthopedics 2002;25:817-9.

31. Muller M, Germann G, Sauerbier M. Minimal invasive screw fixation and early mobilization of acute scaphoid fractures in the middle third: operative technique and early functional outcome. Tech Hand Up Extrem Surg 2008;12:107-13.

32. Shih JT, Lee HM, Hou YT, Tan CM. Results of arthroscopic reduction and percutaneous fixation for acute displaced scaphoid fractures. Arthroscopy 2005;21:620-6.

33. Barton NJ. The late consequences of scaphoid fractures. J Bone Joint Surg Br 2004;86:626-30.

34. Lindström G, Nyström A. Incidence of post-traumatic arthrosis after primary healing of scaphoid fractures: a clinical and radiological study. J Hand Surg Br 1990;15:113.

35. Amadio PC, Berquist TH, Smith DK, Ilstrup DM, Cooney WP 3rd, Linscheid RL. Scaphoid malunion. J Hand Surg Am 1989;14:679-87.

36. Inoue G, Sakuma M. The natural history of scaphoid nonunion. Radiographical and clinical analysis in 102 cases. Arch Orthop Trauma Surg 1996;115:1-4. 